\title{
Survival and the immune response in patients with carcinoma of the colorectum
}

\author{
A. K. HOUSE, AND A. G. WATT \\ From the University Department of Surgery, Queen Elizabeth II Medical Centre and the \\ Royal Perth Hospital, Perth, Western Australia
}

SUMMARY One hundred and seven patients with colorectal carcinoma were examined for their immune response to autologous tumour. Patient leucocyte migration inhibition by tumour antigen, migration inhibition of normal subject's leucocytes by patient's serum, and the assessment of lymphocyte invasion of the tumour specimen were used to measure the immune response. Forty-one and $51 \%$ of patients were immune by leucocyte migration inhibition and tumour invasion by lymphocytes respectively. The immune patients had a survival advantage at three years on Chisquared analysis. Patients whose serum contained a factor that inhibited the migration of leucocytes obtained from normal individuals had a diminished survival prognosis. The donors of these sera had a $50 \%$ chance of dying or of developing recurrent disease. These same individuals represented $30 \%$ of all those tested by this method. The immune patients included the sera donors as a subgroup who, if excluded from the life table analysis, left a group of immune subjects who had very superior survival features.

The prognosis for patients with carcinoma of the colon has remained unchanged for the past two or three decades. Those individuals who can be offered curative surgery have a better survival (Slaney, 1971; Gill and Morris, 1978). In addition, patients with 'early' lesions, Dukes stage A or B, and those who can be offered an anterior instead of an abdominoperineal resection enjoy a survival advantage (Gill and Morris, 1978).

Despite these clinical prognostic observations, the final denominator determining survival is the host tumour interaction. This response is best seen in the tumour invasion profile, which can be used to predict the likely disease progression (Montessori and Donald, 1978). This profile is possibly an expression of tumour factors like their ability to secrete mucus or their histological grade (Grinnell, 1939) and the host immunological reaction to the tumour. Previously we have examined the host immune response to carcinoma of the colon by measuring the lymphocyte invasion of the tumour specimen (Watt and House, 1978) and the leucocyte migration inhibition response of patient's leucocytes to their tumour (House et al., 1975). The present study extends these observations.

Received for publication 26 April 1979

\section{Methods}

\section{PATIENTS}

The subject under study included 107 individuals with colorectal carcinoma on whom a series of 340 leucocyte migration inhibition observations were made. The tumors of these same patients were examined for invasion by lymphocytes. Review of these 107 patients showed there were 29 right, 45 left colonic, and 33 rectal tumours and, among these, 29 were stages Dukes A, 37 Dukes B, and 41 Dukes C. The observation time was from two to six years or until death. The details relating to patient follow-up were collected retrospectively from hospital records, general practitioners, and the Registry of Deaths in Western Australia. The patients' differential white blood cell counts were not regularly available to correlate with the in vitro assays.

Three hundred and forty leucocyte migration inhibition assays were also performed on a control group of 150 patients who were attending the hospital for disease which was not neoplastic or immunological in nature. They were selected to match the patients for blood group, age, severity of operative trauma, and time after surgery at which the tests were performed. 
TUMOUR SPECIMENS

The resected specimen was collected in saline and portions of it prepared as tissue homogenates (House et al., 1975). These homogenates, one of tumour and one of normal mucosa, were used in tissue culture as antigen at $300 \mu \mathrm{g} / \mathrm{ml}$ by protein estimation. A previous trial in which varying antigen concentrations had been used in culture showed that this quantity of antigen did not cause non-specific toxic inhibition of migration. The unused homogenate was stored at $-25^{\circ} \mathrm{C}$ in small aliquots until required.

The remaining portion of the operative specimen was formalin fixed and prepared for routine histological examination.

\section{METHOD OF LEUCOCYTE MIGRATION}

INHIBITION

Twenty millilitres of heparinised venous blood were collected in the first and subsequent postoperative weeks from the patients and matched controls. The majority of the observations were made in the first 12 months after surgery when the patients were most readily available. The collected blood samples were prepared in leucocyte migration inhibition cultures in a manner previously described (House et al., 1975). The complete buffy coat of the collected blood was used in these assays.

The control subjects cell pellet was divided in half and one half incubated in the patient's serum at $37^{\circ} \mathrm{C}$ for 30 minutes and then washed and placed in leucocyte migration cultures.

The culture chambers were arranged so that there were five control cultures containing tissue culture medium (TCM) and $10 \%$ foetal calf serum (FCS) only and 10 test cultures, five containing TCM, $10 \%$ FCS and $300 \mu \mathrm{g} / \mathrm{ml}$ of tumour homogenate, and five containing TCM, $10 \%$ FCS and $300 \mu \mathrm{g} / \mathrm{ml}$ of normal colon extract. This arrangement was used for the tumour patient's leucocytes, the normal subject's leucocytes, and the normal subject's leucocytes after they had been incubated in patient's serum. The cultures were incubated at $37^{\circ} \mathrm{C}$ for 20 hours and the resulting areas of leucocyte migration were measured. The migration index (MI) was calculated from the ratio $\frac{\text { test area }}{\text { control area. }}$ The results of the migration index obtained with the patient's cells in culture with tumour and normal bowel antigen were compared by Student's paired $t$ test. The migration index results for the control subjects were treated similarly and those obtained in the patient group and control subjects were compared by the Mann-Whitney $U$ test. Migration inhibition was considered to indicate immunity to the specific tissue extract used in culture.

QUANTITATIVE ASSESSMENT OF LYMPHOCYTE INFILTRATION AT PERIPHERY OF TUMOUR The prepared section of tumour tissue used in the histological diagnosis of the tumour in 84 patients was examined by high power microscopy. The lymphocytes at the edge of the tumour tissue were counted and grouped as $\geqslant 10$ or $\leqslant 10$ per high power grid field (HPGF). The method used has been reported (Watt and House, 1978). Those patients with counts greater than 10/HPGF were considered immune.

PATIENT SURVIVAL ANALYSIS

The survival of immune and non-immune patients was calculated by the life table method. Survival was considered in six monthly intervals. This data was converted to natural logarithms and the resulting survival curves compared in slope and intercept by an analysis of covariance (Metcalf, 1974). Patient survival at three years was also compared by Chisquared analysis.

\section{Results}

\section{EVALUATION OF LEUCOCYTE MIGRATION} ASSAYS

The migration inhibition assay demonstrated that the patient and control subjects behaved differently when tumour or normal colon antigen were in culture. The tumour patients responded to tumour antigen more often and to a greater level of inhibition than did the control subjects' cells. The difference in

Table 1 Mean migration indices for leucocyte migration inhibition responses

\begin{tabular}{|c|c|c|c|}
\hline \multirow{2}{*}{ Antigen in culture } & \multicolumn{2}{|c|}{ Mean migration index \pm standard deviation } & \multirow{2}{*}{$\begin{array}{l}\text { Statistical difference between } \\
\text { patients and control subjects } \\
\text { Mann-Whitney U-test }\end{array}$} \\
\hline & Tumour patients & Control patients & \\
\hline $\begin{array}{l}\text { Patients } \\
\text { Tumour } \\
\text { Patients } \\
\text { Normal colon }\end{array}$ & $\begin{array}{l}0.79 \\
\pm 0.15 \\
0.82 \\
\pm 0.12\end{array}$ & $\begin{array}{l}0 \cdot 83 \\
\pm 0 \cdot 11 \\
0 \cdot 84 \\
\pm 0 \cdot 10\end{array}$ & $\begin{array}{l}\mathrm{Z}=\mathbf{2 . 1 6 3} \\
\mathrm{P}<0.02 \\
\mathrm{Z}=1.983 \\
P<0.05\end{array}$ \\
\hline
\end{tabular}

Statistical difference (Student's paired $t$ test) between normal colon antigen and tumour antigen when in culture with: tumour patients $t=2 \cdot 94$, $P<0.005$; control patients NS. 
Table 2 Patient survival related to histology and immunity

\begin{tabular}{|c|c|c|c|c|}
\hline & \multicolumn{3}{|c|}{ Differentiated: } & \multirow[t]{2}{*}{ Total } \\
\hline & Well & Mod. & Poor & \\
\hline \multirow[t]{3}{*}{$\begin{array}{l}\text { Immune } \\
\text { Non-immune }\end{array}$} & $\begin{array}{l}22(11) \\
24(7)\end{array}$ & $\begin{array}{ll}17(6) \\
31(17)\end{array}$ & $\begin{array}{l}5(4) \\
8(6)\end{array}$ & $\begin{array}{l}44(21)(5)^{*} \\
63(30)(6)^{*}\end{array}$ \\
\hline & \multicolumn{4}{|c|}{ Neoplasia deaths } \\
\hline & \multicolumn{2}{|c|}{ Category } & No. & $\begin{array}{l}\text { Average survival } \\
\text { (days) }\end{array}$ \\
\hline Immune & \multicolumn{2}{|c|}{$\begin{array}{l}\text { All patients } \\
\text { Dukes } B \text { and } C\end{array}$} & $\begin{array}{l}16 \\
14\end{array}$ & $\begin{array}{l}518 \\
451\end{array}$ \\
\hline Non-immune & \multicolumn{2}{|c|}{$\begin{array}{l}\text { All patients } \\
\text { Dukes B and C }\end{array}$} & $\begin{array}{l}24 \\
22\end{array}$ & $\begin{array}{l}488 \\
440\end{array}$ \\
\hline
\end{tabular}

Number of deaths in parentheses.

*Died from unrelated disease.

the mean migration indices of the two groups of data is statistically different on Mann-Whitney $U$ test. The reaction to normal colon antigen is also statistically different by the same test but this difference is not reflected in the mean values.

The tumour patients leucocytes were inhibited in migration by tumour antigen more frequently and to a greater extent than they were by normal colon antigen. This difference is statistically significant on Student's paired $t$ test. The control subjects leucocytes did not react in this manner. There was no statistical difference between the migration inhibition due to tumour or normal colon antigen (Table 1).

Two criteria for immunity by leucocyte migration inhibition were tried. Firstly, $20 \%$ inhibition was used. This value has most frequently been considered by many laboratories to be diagnostic. Secondly, a stricter criteria of $29 \%$ inhibition was employed. This figure represented the control subjects mean leucocyte migration inhibition to tumour antigen less one standard deviation and the tumour patients response to normal colon antigen less one standard deviation. Both these criteria defined a group of patients whose leucocyte migration was inhibited by tumour antigen in culture so that they could be considered immune.

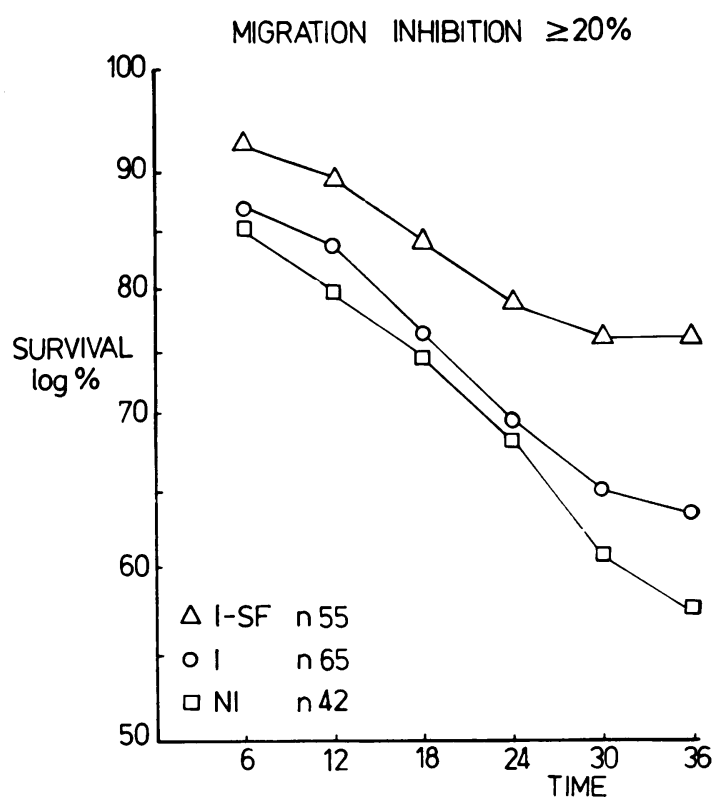

Fig. 1 Log survival curves for immune (O) by $20 \%$ migration inhibition and non-immune $(\square)$ patients. It also shows the survival of the immune group if those with serum factors that inhibit normal cell migration wero removed from the life tables $(\triangle)$. There was a Chi-squared difference in survival at three years between this group $(\triangle)$ and the non-immune patients $(\square)$ $\chi^{2}=3 \cdot 125, \mathrm{P}<0.05$. The curves analysed for covariance did not show differences between the immune (O) and non-immune group but there were differences between the non-immune $(\square)$ and modified immune group $(\triangle)$. Intercepts $F=25 \cdot 30, \mathrm{P}<0 \cdot 01$. Slopes $F=36 \cdot 82$, $\mathbf{P}<0.01$.

IMMUNE PATIENTS AT $20 \%$ OR $29 \%$ INHIBITION OF MIGRATION

Sixty-five $(61 \%)$ patients were immune if $20 \%$ migration inhibition were used to indicate immunity. The life table analysis of these immune and nonimmune subjects make no distinction between the two in survival characteristics (Fig. 1). If a stricte $\mathbf{r}$

Table 3 Compares immune and non-immune group with tumour stage

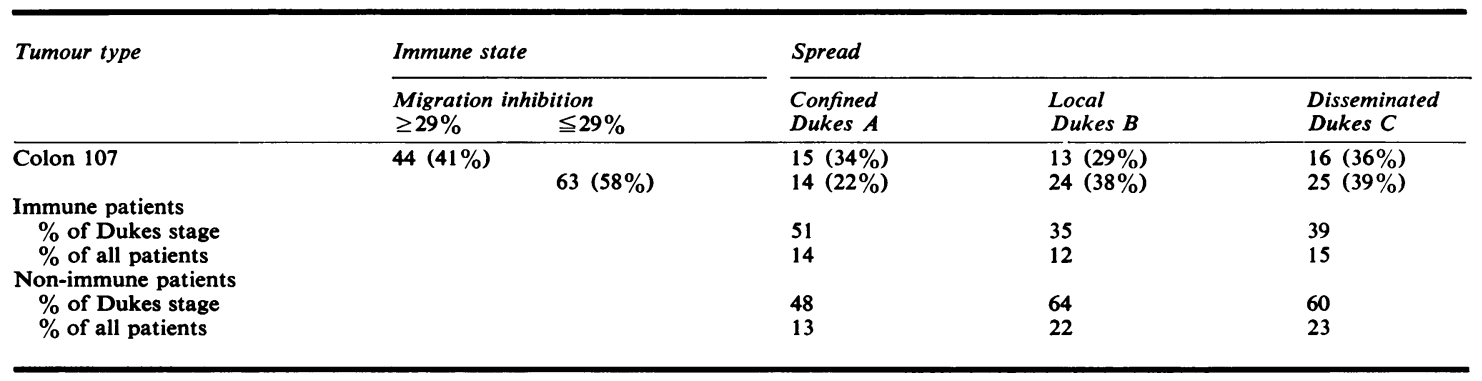


criteria of immunity, $29 \%$ migration inhibition, were adopted $44(41 \%)$ patients were immune. These immune patients are distributed through all Dukes stages and histological grades (Tables 2 and 3). The survival curve analysis shows a difference in slope but not intercept. There is also a Chi-squared survival difference at three years $\chi^{2}=4 \cdot 1437, \mathrm{P}<0.05$ (Fig. 2).

PATIENTS SERUM FACTORS: THEIR

INFLUENCE ON NORMAL SUBJECTS

LEUCOCYTE MIGRATION AND PATIENT

SUR VIVAL

Normal cells incubated in patient serum have altered migration functions. Some sera spontaneously inhibited migration and others conferred passive immunity so that the presence in culture of the appropriate tumour antigen inhibited migration. Sixty-nine patients had serial examinations of their sera, 42 of these inhibited migration spontaneously and 21 conferred passive immunity. The former group contained 22 immune and 20 non-immune patients. The donors of sera that contained leucocyte inhibitory properties had a high risk $(50 \%)$ of developing recurrent disease or of dying of their disease. The donors of sera that conferred passive immunity were not at this risk but their sera sometimes altered to inhibit migration spontaneously (Table 4).

The effect on survival of the migration inhibiting serum factor is demonstrated in Fig. 1. There were 10 immune patients, determined by $20 \%$ migration inhibition who were at risk because their serum contained this factor. Their removal from the life table analysis resulted in a survival curve which on analysis was both different in slope and intercept. This curve appeared to be becoming more favourable at three years and these immune patients, at this time, survived significantly better than the non-immune individuals on Chi-squared testing: $\chi^{2}=3 \cdot 125$, $\mathbf{P}<0.05$.

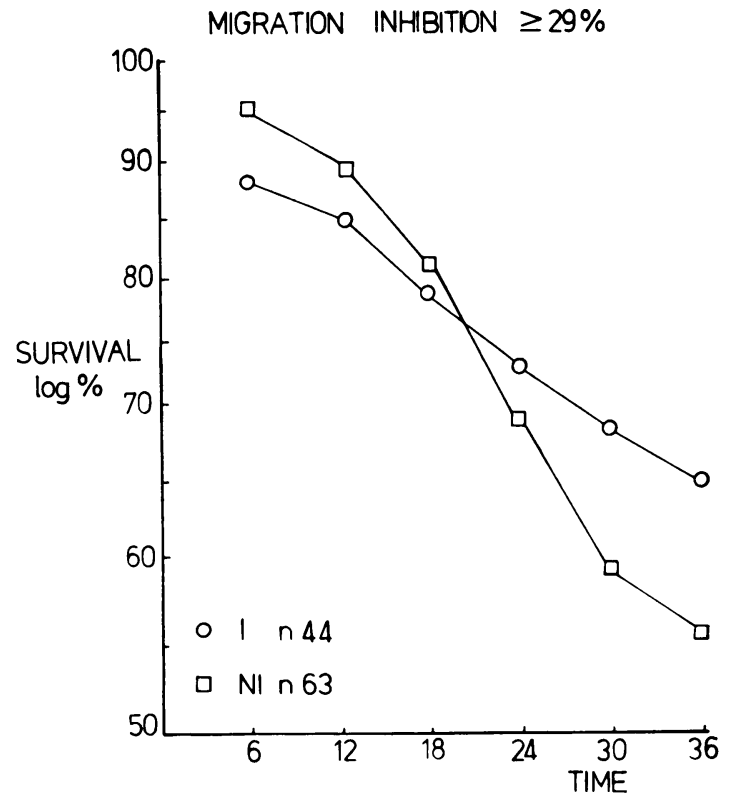

Fig. 2 Log survival curves for immune (O) and non-immune patients $(\square)$ when leucocyte migration of $29 \%$ by tumour antigen was recorded in the immune patients. The curves analysed for covariance showed differences in slope $F=15.91, \mathrm{P}<0.01$ but not intercept $F=1 \cdot 182, \mathrm{P}>0 \cdot 05$. The survival at three years was different on Chi-squared testing: $\chi^{2}=4 \cdot 1437$, $\mathbf{P}<0.05$.

\section{TUMOUR LYMPHOCYTE INFILTRATION AND} SUR VIVAL

There were $43(51 \%)$ immune and 41 non-immune patients by tumour invasion of 10 or more lymphocytes per high power grid field. The life table analysis and survival curves were significantly different in slope and intercept. Three year survival was also significantly different on Chi-squared testing: $\chi^{2}=3.583, \mathrm{P}<0.05$ (Fig. 3).

Table 4 Control leucocyte migration inhibition after incubation in patients' sera

\begin{tabular}{|c|c|c|c|c|}
\hline \multirow[t]{2}{*}{ Neoplasm site } & \multirow[t]{2}{*}{ No. of patients' sera } & \multicolumn{3}{|c|}{$\begin{array}{l}29 \% \text { inhibition of migration of control leucocytes by } \\
\text { patients' serum incubation: }\end{array}$} \\
\hline & & $\begin{array}{l}\text { Alone } \\
\text { A }\end{array}$ & & $\begin{array}{l}+ \text { Tumor antigen } \\
B\end{array}$ \\
\hline \multicolumn{5}{|l|}{ Colon } \\
\hline Patients & 69 & $42 \quad 61 \%$ & F13F & $2136 \%$ \\
\hline Immune & 27 & $22(10)$ & F 7F & 12 \\
\hline Non-immune & 42 & $20(11)$ & $\mid F 6$ F & 12 \\
\hline No. of observations & 204 & & & \\
\hline \multicolumn{5}{|l|}{ Death/recurrence } \\
\hline + ve Patients & $30 \%$ & $2150 \%$ & & 0 \\
\hline
\end{tabular}

Numbers in parentheses developed recurrence or died.

Numbers in box became - ve or were associated with concurrent + ve in column $B$. 


\section{TUMOUR LYMPHOCYTES}

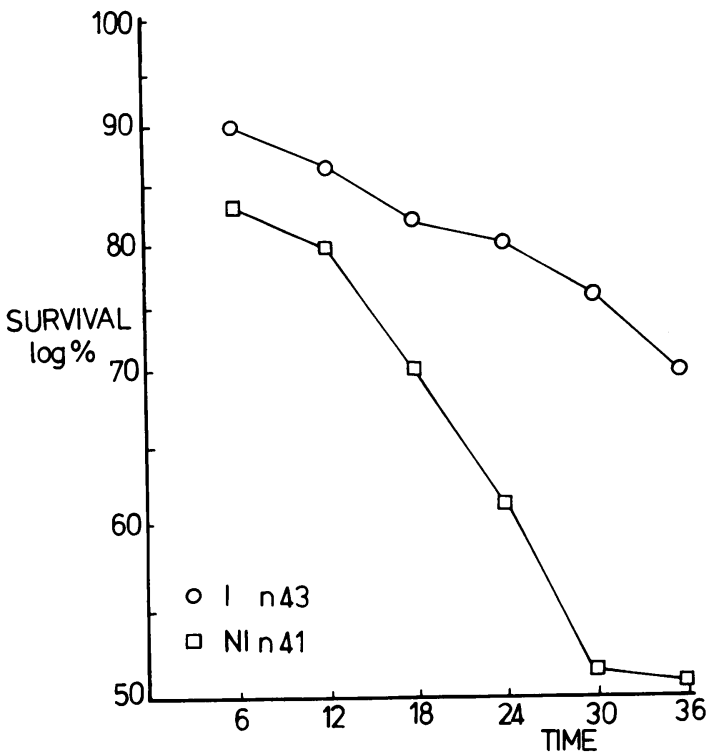

Fig. 3 Log survival curves for immune (O) and non-immune $(\square)$ patients measured by lymphocyte invasion of the patient's tumour. The curve analysis for covariance was different in slope $F=42.68, \mathrm{P}<0.01$ and intercepts $F=12.89, \mathrm{P}<0.01$. The survival at three years was different on Chi-squared testing: $\chi^{2}=3 \cdot 583$, $\mathbf{P}<0.05$.

\section{Discussion}

Leucocyte migration inhibition has been used to define immunity in a number of pathological conditions (Sorborg and Bendixen, 1967; Elias and Elias, 1975; Imai et al., 1977). These authors have all used $20 \%$ migration inhibition to indicate immunity. The mean response of the patients to normal colon antigen and the control subjects to both tumour and normal colon antigen indicated that all those tested were not immune to these antigens. However, the standard deviation from the mean placed some of the recorded values within the $20 \%$ inhibition immune range, which suggested that the cell donor may have been immune to the particular test antigen.

There were a number of alternative explanations for this immune response. Particulate matter in the culture system may have been a cause of false positive leucocyte migration inhibition, but others have shown that particulate antigens are more effective in the migration assay than soluble ones (Gorski, 1974). In addition, the reaction will not function in the absence of lymphocytes (Fimmel, 1975), which would suggest that the response is specifically directed toward the specific antigen.

Common antigens between the tumour and normal colon preparations of cellular or contaminant bacterial origin may have been responsible for some false responses. However, care was taken in the antigen preparation to minimise these possibilities and they seem unlikely, for within the tumour group few patients responded to normal colon antigen. This was verified by the clear statistical distinction between the mean migration inhibition values obtained when the results for the two antigens were compared: $P<0.005$. There also was a statistical difference between the results of the patient and control subjects when both the tests with tumour and colon antigen were analysed. However, the difference between the two groups with colon antigen was small and so was probably not of biological significance.

Additional explanation is proffered in the control subject tests. There was no statistically significant difference between the control subject responses to tumour or normal colon antigen. The occasional positive normal subject immune response could have been due to a number of variants, allogeneic differences between the test antigens and the control individual, presensitisation to possible contaminant bacterial antigens, or an occult neoplasm in the leucocyte donor. Those few subjects who have had positive responses are being screened for the latter possibility. Cochrum et al. (1977) have shown that the first explanation is possible. These authors demonstrated that leucocyte migration inhibition by HLA antigens correlates with mixed lymphocyte culture defined allogeneic differences.

The mean migration index for the tumour patients to their tumour antigen was within the immune range. Sixty-five $(61 \%)$ of our patient group were immune at $20 \%$ of migration inhibition by this antigen. This percentage of immune patients is similar to that reported by Guillou and Giles (1973) but is lower than that reported by Elias and Elias (1975). This level of migration inhibition as a criteria for immunity in this study was not discriminatory without qualification. The survival of this immune group was not significantly different from the non-immune group.

Those patients who were immune by migration inhibition of $29 \%$ by tumour antigen were clearly distinct from the control subjects. The immune group of patients was reduced to $44(41 \%)$. This number was similar to that previously reported by us (House et al., 1975) and similar to that found in carcinoma of the breast (Stein at al., 1976). The stricter criteria were also discriminatory in survival terms. This immune group of patients enjoyed a survival advantage that was statistically significant at three years.

The immune patients in this study were found to 
have tumours that spanned all the histological grades. However, there were proportionally more patients in the immune group who had welldifferentiated neoplasms. This may have been a reflection of a well-developed host tumour interaction but the relatively poor crude survival $(50 \%$ mortality) among these individuals might suggest the contrary. At the time of review there were 16 $(39 \%)$ deaths due to carcinoma in the immune subjects and $24(38 \%)$ in the non-immune group. The average survival for the dead was 518 and 488 days respectively.

The immune patients were found among those grouped in each of the Dukes stages. The total number contained immune and non-immune subjects in equal proportions of stage $\mathrm{A}$, but $50 \%$ and $25 \%$ more non-immune stage $\mathrm{B}$ and $\mathrm{C}$ individuals. However, within the immune group, there was a greater percentage of stage A patients $(51 \%)$ than those classified Dukes C (39\%). This compares with similar percentages of each stage in the non-immune subjects. This distribution was similar to that found by Rao et al. (1977) but at variance with that reported by Jubert et al. (1977) and Elias et al. (1977).

The results in this study suggested that it is possible to passively confer immunity on normal subjects cells by incubating them in patient's serum. The donor of such a serum did not appear to suffer a detrimental consequence. However, the donor of a serum that spontaneously inhibited the migration of normal leucocytes in the absence of the immunising tumour antigen did have an altered prognosis. These donors had a $50 \%$ chance of developing recurrence or dying of their disease. Others have reported that serum factors had an immune suppression effect in carcinoma of the breast (Whittaker et al., 1971), colorectal cancer (Guillou and Giles, 1973), and melanoma (Cochran et al., 1976). More recently, Hoffken et al. (1978) have shown a relationship between survival and circulating immune complexes. Serum factors did influence survival in patients defined as immune by leucocyte migration inhibition of $20 \%$. Subtraction of a defined serum factor associated immune sub-group from the $20 \%$ inhibition immune patients left the remaining patients with a considerably better survival prognosis at three years.

An inflammatory infiltrate in and around the tumour and sinus histocytosis in the draining lymph nodes have been suggested as morphological manifestations of host resistance. These infiltrates have been associated with improved prognosis in carcinomata of the breast (Anastassides and Pryce, 1966), stomach (Black et al., 1971) and colon (Murray et al., 1975). The findings in this study confirm these results. Lymphocyte infiltration at the periphery of the tumour was associated with a statistically better prognosis at three years and the survival curve suggested that this would be maintained in later years.

We are grateful to the Cancer Council of Western Australia for financial support and to our colleagues for allowing study of patients in their care. We also thank Professor B. N. Catchpole for his interest and Professor Sir John Loewenthal for making the facilities of his department available to us during the construction of the manuscript. We are also extremely grateful to Professor J. M. Little for his helpful advice concerning the statistical evaluation of the data. We thank our laboratory assistant, Margaret Gruar, and the staff of the Records Departments of the Sir Charles Gairdner and Royal Perth Hospitals.

\section{References}

Anastassiades, O., and Pryce, D. M. (1966). Immunological significance of the morphological changes in lymph nodes draining breast cancer. British Journal of Cancer, 20 239-249.

Black, M. M., Freeman, C., Mork, T., Harvei, S., and Cutler, S. J. (1971). Prognostic significance of microscopic structure of gastric carcinomas and their regional lymph nodes. Cancer, 27, 703-711.

Cochrum, K. C., Salvatierra, O., Cullen, B. L., Perkins, H. A., Hanes, D. M., and Fudenberg, H. H. (1977) Leucocyte migration inhibitory factor (LIF) as an indicator of mixed lymphocyte culture (MLC) reactivity. Transplantation Proceedings, 9, 97-101.

Cochran, A. J., Mackie, R. M., Ross, C. E., Ogg, L. J., and Jackson, A. M. (1976). Leukocyte migration inhibition by cancer patients' sera. International Journal of Cancer, 18, 274-281.

Elias, E. G., and Elias, L. L. (1975). Autologous leucocyte migration in human malignancies. Cancer, 36, 1393-1398.

Elias, E. G., Elias, L. F., Didolkar, M. S., and Hebel, J. R. (1977). Cellular immunity in patients with colorectal adenocarcinoma measured by autologous leukocyte migration inhibition. Cancer, 40, 687-692.

Fimmel, P. J. (1957). Studies on leukocyte migration inhibition: The role of $E$ Rosette-forming cells in specific antigen-induced inhibition of migration. Journal of Immunology, 115, 135-138.

Gill, P. G., and Morris, P. J. (1978). The survival of patients with colorectal cancer treated in a regional hospital. British Journal of Surgery, 65, 17-20.

Gorski, A. J. (1974). Superiority of corpuscular BCG to soluble PPD antigen in the leucocyte migration assay. Clinical and Experimental Immunology, 18, 149-153.

Grinnel, R. S. (1939). The grading and prognosis of carcinoma of the colon and rectum. Annals of Surgery, 109, $500-533$.

Guillou, P. J., and Giles, G. R. (1973). Inhibition of leucocyte migration by tumour-associated antigens of the colon and rectum. Gut, 14, 733-738.

Hoffken, K., Meredith, I. D., Robins, R. A., Baldwin, R. W., Davies, C. J., and Blamey, R. W. (1977). Circulating immune complexes in patients with breast cancer. British Medical Journal, 2, 218-220.

House, A. K., Wisniewski, S., and Woodings, T. L. (1975). 
Immunity in colonic tumor patients after operation; determination by leukocyte-migration inhibition. Diseases of the Colon and Rectum, 18, 100-106.

Imai, K., Hosokawa, Y., Abe, H., Yachi, A., and Wada, T. (1977). Inhibition of leucocyte migration by potassium chloride extracts of human tumor cells in ascities and from tissues. Gann: Japanese Journal of Cancer Research (Tokyo), 68, 581-587.

Jubert, A. V., Talbott, T. M., Mazier, W. P., MacKeigan, J. M., Campos, M. M., Benjamin, H. G., Muldoon, J. P., Ferguson, J. A., Maycroft, T. M., and Bowman, H. E. (1977). Correlation of immune responses with Dukes classification in colorectal carcinoma. Surgery, 82, 452-459.

Metcalf, W. (1974). Analysis of cancer survival as an exponential phenomenon. Surgery, Gynecology and Obstetrics, 138, 731-740.

Montessori, G.A., and Donald, J. C. (1978). Invasion profile of colorectal carcinoma. Diseases of the Colon and Rectum, 21, 26-28.

Murray, D., Hreno, A., Dutton, J., and Hampson, L. G. (1975). Prognosis in colon cancer. A pathologic reassessment. Archives of Surgery, 110, 908-913.
Rao, B., Wanebo, H. J., Pinsky, C., Stearns, M., Jr, and Oettgen, H. F. (1977). Delayed hypersensitivity reactions in patients with carcinoma of the colon and rectum. Surgery, Gynecology and Obstetrics, 144, 677-681.

Slaney, G. (1971). Results of treatment of carcinoma of the colon and rectum. In Modern Trends in Surgery, 3, pp. 69-89. Edited by W. T. Irvine. London: Butterworths.

Soborg, M., and Bendixen, G. (1967). Human lymphocyte migration as a parameter of hypersensitivity. Acta Medica Scandinavica, 181, 247-256.

Stein, J. A., Adler, A., Efraim, S. B., and Maor, M. (1976). Immuno-competence, immunosuppression and human breast cancer. 1. An analysis of their relationship by known parameters of cell-mediated immunity in welldefined clinical stages of disease. Cancer, 38, 1171-1187.

Watt, A. G., and House, A. K. (1978). Colonic carcinoma. A quantitative assessment of lymphocyte infiltration at the periphery of colonic tumors related to prognosis. Cancer, 41, 279-282.

Whittaker, M. G., Rees, K., and Clark, C. G. (1971). Reduced lymphocyte transformation in breast cancer. Lancet, 1 , 892-893. 